\title{
Monitoring insect pollinators and flower visitation: the effectiveness and feasibility of different survey methods
}

Article

Accepted Version

O'Connor, R. S., Kunin, W. E., Garratt, M. P. D., Potts, S. G., Roy, H. E., Andrews, C., Jones, C. M., Peyton, J., Savage, J., Harvey, M., Morris, R. K. A., Roberts, S. P. M., Wright, I., Vanbergen, A. J. and Carvell, C. (2019) Monitoring insect pollinators and flower visitation: the effectiveness and feasibility of different survey methods. Methods in Ecology and Evolution, 10 (12). pp. 2129-2140. ISSN 2041-210X doi: https://doi.org/10.1111/2041-210X.13292 Available at https://centaur.reading.ac.uk/86127/

It is advisable to refer to the publisher's version if you intend to cite from the work. See Guidance on citing.

To link to this article DOI: http://dx.doi.org/10.1111/2041-210X.13292

Publisher: Wiley

All outputs in CentAUR are protected by Intellectual Property Rights law, including copyright law. Copyright and IPR is retained by the creators or other copyright holders. Terms and conditions for use of this material are defined in the End User Agreement. 


\section{www.reading.ac.uk/centaur}

\section{CentAUR}

Central Archive at the University of Reading

Reading's research outputs online 


\section{Methods in Ecology and Evolution}

DR RORY SEAN O'CONNOR (Orcid ID : 0000-0001-7633-4304)

DR MICHAEL PAUL DOUGLAS GARRATT (Orcid ID : 0000-0002-0196-6013)

Article type : Research Article

Editor : Luisa Carvalheiro

\section{Monitoring insect pollinators and flower visitation: the effectiveness and feasibility of different survey methods}

Corresponding author mail :- r.oconnor@reading.ac.uk

Rory S. O’Connor ${ }^{1,2^{*}}$, William E. Kunin ${ }^{2}$, Michael P. D. Garratt ${ }^{1}$, Simon G. Potts ${ }^{1}$, Helen E. Roy $^{3}$, Christopher Andrews ${ }^{4}$, Catherine M. Jones ${ }^{2}$, Jodey Peyton ${ }^{3}$, Joanna Savage ${ }^{3}$, Martin Harvey $^{3}$, Roger K.A. Morris ${ }^{5}$, Stuart P.M. Roberts ${ }^{1}$, Ivan Wright ${ }^{6}$, Adam J. Vanbergen ${ }^{4,7}$ and Claire Carvell ${ }^{3}$.

${ }^{1}$ Centre for Agri-Environmental Research, School of Agriculture, Policy and Development, University of Reading, Reading, RG6 6AR, UK;

${ }^{2}$ The Faculty of Biological Sciences, University of Leeds, Leeds, LS2 9JT, UK;

${ }^{3}$ Centre for Ecology \& Hydrology, Maclean Building, Crowmarsh Gifford, Wallingford, OX10 8BB, UK;

${ }^{4}$ Centre for Ecology \& Hydrology, Bush Estate, Penicuik, Midlothian, EH26 0QB, UK;

${ }^{5} 241$ Commonside East, Mitcham, Surrey CR4 1HB, UK;

${ }^{6}$ Shotover Wildlife, Horspath, Oxford, OX33 1SB, UK;

${ }^{7}$ Agroécologie, AgroSup Dijon, INRA, Univ. Bourgogne Franche-Comté, F-21000 Dijon, France.

This article has been accepted for publication and undergone full peer review but has not been through the copyediting, typesetting, pagination and proofreading process, which may lead to differences between this version and the Version of Record. Please cite this article as doi: 10.1111/2041-210X.13292

This article is protected by copyright. All rights reserved. 


\section{Summary}

1. The status of pollinating insects is of international concern, but knowledge of the magnitude and extent of declines is limited by a lack of systematic monitoring. Standardised protocols are urgently needed, alongside a better understanding of how different methods and recorders (data collectors) influence estimates of pollinator abundance and diversity.

2. We compared two common methods for sampling wild pollinating insects (solitary bees, bumblebees and hoverflies), pan traps and transects, in surveys of $1 \mathrm{~km}$ countryside squares (agricultural and semi-natural habitats) and flowering crop fields across Great Britain, including the influence of local floral resources (nectar sugar availability or crop flower density) on the insects sampled. Further, we compared the performance of recorders with differing expertise (non-specialist research staff, taxonomic experts and non-expert volunteers) in applying methods.

3. Pan traps and transects produced compositionally distinct samples of pollinator communities. In the wider countryside, pan traps sampled more species of solitary bee and hoverfly. In flowering crops, transects recorded greater numbers of individual bumblebees, but fewer species.

4. Across all taxonomic groups and countryside and crop samples, transects generally had lower rates of species accumulation per individual collected than pan traps. This demonstrates that differences between methods in estimating richness are not due to sampling effort alone. However, recorders possessing greater taxonomic expertise can produce species accumulation data from transects that is almost commensurate with pan trapping.

5. The abundance and species richness of pollinators (except solitary bees) on transects in the wider countryside was positively related to the availability of estimated nectar sugar. In crops, pollinator abundance responses to flower densities were idiosyncratic according to crop type, but overall the response was positive and negative for transects and pan traps, respectively.

6. Given these taxonomic and context-specific differences in method performance, we assess their suitability for monitoring pollinating insect communities and pollination services. We discuss the relevance of these findings within the context of achieving standardised, large-scale monitoring of pollinating insects.

Key-words: Pollinator monitoring, abundance, diversity, bees, hoverflies, pan traps, transects, expertise

This article is protected by copyright. All rights reserved. 


\section{Introduction}

There is international concern about declines in the diversity and distribution of insect pollinators and the consequences for pollination services (Potts et al., 2016). Research is increasingly demonstrating how land-use change, pesticides, climate change, invasive nonnative species, pests and disease may act, and interact, to cause declines in pollinating insects (Vanbergen et al., 2013). However, evidence is incomplete and important gaps remain with respect to the magnitude, geographic and taxonomic extent of these declines (Potts et al., 2016). For example, our understanding of the population status and trends in abundance and diversity of pollinating insects is severely limited by a worldwide lack of standardized, longterm and large-scale data (Lebuhn et al., 2013). This creates an urgent need for monitoring and protocols that accommodate broad taxonomic and geographic coverage, account for potential biases in the data and generate adequate sample sizes; all whilst remaining cost effective.

The most important providers of pollination services globally are insects, particularly bees and some flies (e.g. hoverflies) (Potts et al., 2016). Current best evidence for the status of wild bees and hoverflies comes from records of species occurrence collected in national and global biodiversity databases. In Great Britain (GB), records collated by the Bees, Wasps and Ants Recording Society and the Hoverfly Recording Scheme have allowed unparalleled insights into the status and distributional changes of bees and hoverflies in GB (Carvalheiro et al., 2013; Powney et al., 2019). To our knowledge such verified long-term occurrence data for wild bees and hoverflies exist only for GB, the Netherlands, Belgium (Carvalheiro et al., 2013) and bumblebees in the USA (Cameron et al., 2011). These data are collected using unstandardized or semi-standardized protocols (Isaac \& Pocock, 2015) and changes in recording intensity, taxonomic ability and sampling strategies mean sources of bias have not been consistent over time. Critically, occurrence records provide no standardized estimates of abundance, which are fundamental to understanding changes in population size and the links between pollinators and pollination services (Potts et al., 2016). Identifying the best approaches for pollinator monitoring is crucial to reduce these limitations.

Different methods for sampling pollinating insects are associated with different outputs and challenges with regard to taxonomic coverage and implementation. Direct observations (transects and observation plots) and pan traps (sampling within painted water-filled bowls) 
are the most commonly used methods (Westphal et al., 2008). Transects and timed focal floral observations are straightforward to conduct and can generate data on insect-plant interactions but depend on the expertise of the observer (Sutherland, Roy, \& Amano, 2015) and may be biased towards more conspicuous species (Dennis et al., 2006). Pan traps tend to sample more species of bee than other standardized methods (Westphal et al., 2008), are independent of observer expertise and are recommended by the Food and Agriculture Organisation (FAO) for monitoring bees in agricultural habitats (LeBuhn et al. 2016). However, pan trap efficacy may be biased because certain taxa (e.g. social bees) may be less likely to be caught and effects of local floral resource density on catches are not well understood (Cane, Minckley, \& Kervin, 2000; but see Wood, Holland, \& Goulson, 2015). Similarly, using non-expert volunteers, or 'citizen scientists', presents an opportunity to collect large amounts of data and engage a wide range of individuals in wildlife recording. However these benefits potentially trade-off against the reduced taxonomic resolution that these volunteers can typically gather and data accuracy (Roy et al., 2016), which is required to address ecological questions concerning the diversity of wild pollinators.

We compared the potential of pan traps and transects for surveying pollinating insects in a) the wider countryside and b) flowering crop fields in 38 sites across GB. Furthermore, in the wider countryside we explored the effect of recorder expertise on the nature and accuracy of data collected using transects and floral observation plots. Thereafter, we outline options for the development of protocols for monitoring pollinator abundance and diversity to facilitate the production of long-term, standardised national and international datasets in accord with international science and policy needs identified by the Intergovernmental Science-Policy Platform on Biodiversity and Ecosystem Services (Potts et al., 2016).

\section{Methods}

\section{Wider countryside surveys}

We tested three commonly used methods for sampling bees and hoverflies (Westphal et al., 2008; O’Connor et al., 2016);

i) Pan traps: a triplet of plastic bowls (350ml capacity; Salbert, Item Number: 92012A500) sprayed with UV fluorescent paint (1 x white, 1 x yellow, 1 x blue; Sparvar "Leuchtfarbe") with each bowl containing $100 \mathrm{ml}$ of water plus a drop of 
unscented detergent to break surface tension. Each triplet (hereafter station) was fixed to a wooden stake using wire supports and set at the average height of flowers or other surrounding vegetation or secured to the ground in very short vegetation or bare ground.

ii) Insect visitation transects: Five transect sections, each $200 \mathrm{~m}$ in length and following a linear route, were walked at a slow pace for between 12-15 minutes allowing for variation in transect terrain. All insects seen visiting flowers were recorded within a $1 \mathrm{~m}^{3}$ sampling box ahead and to the side of the recorder and assigned to one of the following taxonomic groups: bumblebees, honeybees, solitary bees (including primitively eusocial species) and hoverflies. Individual insects were recorded only once. Where species level identifications were required (see below) individuals were netted, placed in a labelled tube and frozen for later identification, unless they could be readily identified in situ. Time spent handling insects for identification was not included in the transect time.

iii) Floral observation plots: a defined area observed for a set time to record insect flower visitors. Plots of 50 x $50 \mathrm{~cm}$ were observed for 10 minutes for insect flower visitation on a focal plant species, insects were observed and recorded once and classified into taxonomic groups, as described above (without specimen identification). Focal plant species on a site were selected from a list of 25 nationally common flowering plants (Table S1) or, if not present, then a locally abundant species. The plant species and number of floral units within each plot were recorded.

The wider countryside surveys used a one-day protocol to sample within a $1 \mathrm{~km}$ square, compatible with existing biodiversity monitoring schemes in GB (e.g. Pescott et al., 2015). Fourteen $1 \mathrm{~km}$ grid squares (Brtish national grid) were sampled across GB (Figure 1a; England $=6$; Scotland $=6$; Wales $=2)$ with half the squares dominated $(>50 \%)$ by seminatural land cover and half dominated by agricultural land cover (arable, horticulture or improved grassland collectively). In each square, we situated five $200 \mathrm{~m}$ transects and five pan trap stations at approximately $200 \mathrm{~m}$ intervals on a diagonal line bisecting the square (Figure 1b), typically following boundary features or, where accessible, following tractor lines within cropped fields or edges of grass fields with livestock.

This article is protected by copyright. All rights reserved. 
Pan trap stations were deployed at the start of each transect (Figure 1b) and left exposed for 6-7 hours (depending on terrain and time taken to complete the other methods) between 10:00 and 16:00. After pan trap deployment, each $200 \mathrm{~m}$ transect section was walked to record insect flower visitors. For each section, available floral resources were quantified. The number of floral units (flower heads, umbels or spikes) of $\geq 5$ most common flowering plant species was also recorded on a 5-point ordinal scale: (1) 1-2, (2) 2-30, (3) 31-300, (4) 3013,000 , (5) $>3,000$. To standardise nectar availability per transect, the total amount of available nectar sugar was estimated for each recorded flowering plant species as $\mu \mathrm{g}$ sugar produced in 24hrs per floral unit (following Baude et al., 2016); see supplementary material). We multiplied this value by the median coverage of each species for categories 1-4 and by 3001 for category 5 and converted it to an estimate of nectar availability per $\mathrm{m}^{2}$ for each transect (by dividing this product by 200). Due to some extreme estimates of flower density we imposed a maximum limit of $20,000 \mu \mathrm{g}$ sugar per $\mathrm{m}^{2}$ per $24 \mathrm{hrs}$. Two 10 -minute focal floral observations per site were also conducted during each sampling day. Each site was sampled once during four sampling rounds in 2015: 1) 27 April-10 May, 2) 1-14 June, 3) 619 July, 4) 17-30 August.

To explore the effect of recorder expertise on the data collected, we classified recorders according to their degree of expertise in field surveys and recognising pollinating insects: (i) non-specialist research staff - employees of universities or research institutes with prior experience of surveying and identifying insects and plants to at least broad group levels; (ii) taxonomic experts - volunteer or professional entomologists who submit records to existing biological recording schemes possessing a high level of expertise in collecting and identifying at least one broad taxonomic group to species level; (iii) non-expert volunteers members of the public who partake in citizen science projects possessing varying levels of familiarity with pollinator identification or ecological surveys. All recorders conducted transects, volunteers and researchers conducted focal observations, but only researchers conducted pan traps. All recorders followed the same protocol for each method and were provided with identification guides for broad insect groups and focal plant species. Research staff and experts collected data to species resolution as far as possible, whereas non-experts only classified insects into broad groups.

This article is protected by copyright. All rights reserved. 
All sites were surveyed by research staff; taxonomic experts visited only the sites in England and Wales and non-expert volunteers were restricted to rounds three and four, surveying on the same days as the research staff. Research staff and volunteers undertook transects within 15 minutes of each other and focal observations in parallel on the same patches of flowers. Fifty-five site visits were achieved by research staff, 25 by taxonomic experts and 17 by volunteer non-experts (Table S2).

\section{Flowering crop surveys}

To compare pollinator survey methods in crops, pan trapping and transects were carried out simultaneously in dessert apples (Malus domestica, variety Cox's Orange Pippin), strawberries (Fragaria X ananassa, mixed varieties) and field beans (Vicia faba, variety Wizard) in the spring and summer of 2011(Garratt \& Potts, 2011). We used eight apple orchards in Kent, eight strawberry fields in Yorkshire and eight field bean fields in Oxfordshire and Berkshire (Figure 1a), with three sampling rounds carried out during strawberry and field bean flowering and two during apple bloom. Sampling plots contained two $150 \mathrm{~m}$ sampling transects, divided into three $50 \mathrm{~m}$ sections and a pan trap station was placed at the end of each section, giving six pseudo-replicates of each method per field (Figure 1c). Transects were at least $25 \mathrm{~m}$ apart and from the field edge (Figure 1c) and each $50 \mathrm{~m}$ section was walked for 10 minutes at a steady pace. Pan traps were as specified above for wider countryside, but used $460 \mathrm{ml}$ bowls, left out for 24 hours in apples and strawberries, and 7-10 hrs in field beans. Apple flower densities were counted within 1 x $1 \mathrm{~m}$ quadrats held against trees at head height, whereas for strawberries a 1 × $2 \mathrm{~m}$ area was assessed. Field bean flowering stems were counted within a 1 x $2 \mathrm{~m}$ area, and multiplied by the mean flower counts on 5 randomly-chosen stems.

\section{Survey conditions and identification}

All surveys were carried out between 10:00-16:00 in dry weather, with light winds $\left(<29 \mathrm{~km} / \mathrm{h}\right.$, Beaufort 5), and where minimum temperatures exceeded $13^{\circ} \mathrm{C}$ if $<50 \%$ cloud cover, or $15^{\circ} \mathrm{C}$ if $>50 \%$ cloud cover (although $11^{\circ} \mathrm{C}$ or $13^{\circ} \mathrm{C}$ was allowed for some upland locations or visits in April). Collected bee and hoverfly specimens were stored in $70 \%$ ethanol for identification to species level by expert taxonomists and archived in $99 \%$ ethanol.

This article is protected by copyright. All rights reserved. 


\section{Analysis}

All analyses were performed using R version 3.3.2 (R Core Team, 2016).

\section{Similarity of pan trap and transect samples of pollinator communities}

Data were summarized at the site ( $1 \mathrm{~km}$ square or crop field) level to demonstrate typical sample sizes achieved by the two methods and by the different recorder groups across the four focal insect groups (Tables 1 and 2; Tables S3 and S4).

We assessed the degree of dissimilarity (Morisita-Horn abundance-based dissimilarity index) between the pollinator (bees and hoverflies identified to species) communities sampled by research staff using pan traps and transects in the wider countryside dataset and each flowering crop dataset (apple, strawberry and field bean separately). To determine if the pan trap and transect methods produced significantly dissimilar assemblages we used permutational ANOVAs (R: vegan: adonis) against random permutations of the original data (countryside $=999 ; \mathrm{FC}=255$ for each crop dataset) (Oksanen et al., 2015). Data for the wider countryside semi-natural dominated site in Wales were excluded due to too few records. Non-metric multidimensional scaling (NMDS) was used to visualize dissimilarity between sampling methods based on Mortista-Horn dissimilarity (R: vegan: MetaNDMS; Oksanen et al., 2015).

\section{The effects of sampling effort and recorder expertise on estimates of species richness}

We used species accumulation curves to understand the influence of sampling effort on the efficacy of methods and recorders to produce species richness estimates given their different modes of action and inherent biases. The number of individuals sampled is the basic currency with which species richness estimates between samples or datasets can be compared. Using the iNEXT package in R (Hsieh, Ma, \& Chao, 2019), we plotted individual-based species accumulation curves that show interpolated species richness (per cumulative individual sampled) up to the total sample size and thereafter extrapolated species richness. Curves were plotted for pan traps and transects using samples amalgamated across the dataset for each broad taxonomic group in the wider countryside dataset, for solitary bees in apples, bumblebees in strawberries and bumblebees and solitary bees in field beans. Further, for a subset of the wider countryside data covering seven sites (four with samples for all four sampling rounds, one for the $2^{\text {nd }}, 3^{\text {rd }}$ and $4^{\text {th }}$ sampling rounds and two for the first two 
sampling rounds, totally 23 sampling visits) individual-based species accumulation curves were plotted for bumblebees, solitary bees and hoverflies to compare pan traps with transects conducted by either researchers or taxonomic experts.

Correlation analyses (Spearman's or Kendall's rank) were used to compare estimates of bumblebee, solitary bee, hoverfly and honeybee abundance from transects walked by research staff and non-expert volunteers (17 site visits with corresponding data) and from parallel floral observation plots.

\section{Per sampling unit differences between pan traps and transects}

Generalized linear mixed models (GLMMs) were used to test for differences between pan traps and transects at the sampling unit level (individual pan trap station or corresponding transect section), along with the effects of local floral resources and other covariates, using the datasets for bumblebees, solitary bees and hoverflies generated by research staff (honeybee numbers were insufficient). Models were fitted and selected using the glmmadmb package (Skaug et al., 2015) which allows zero-inflated models, although poisson or negative binomial errors were appropriate for all models. Final models were selected by stepwise elimination of non-significant variables using log-likelihood tests (Zuur, Hilbe, \& Ieno, 2013). Final models were also run with the lme4 package (Pinheiro et al., 2015) to check the agreement of model fits between packages. In every instance they were comparable, giving the same qualitative results with only slight differences in parameter estimates. The lsmeans package (Lenth, 2016) was used to calculate least square means and marginal effects plots from lme4 output were produced using the SJPlot package (Lüdecke, 2017).

For the abundance and species richness of bumblebees, solitary bees and hoverflies sampled on the wider countryside surveys, initial model predictors included sampling method, sampling round, country (England and Wales were amalgamated into one level due to low replication for Wales), log estimated nectar sugar availability per transect ( $\mu \mathrm{g}$ per 24 hours), maximum daytime temperature $\left({ }^{\circ} \mathrm{C}\right)$ from the nearest UK MET office recording station and dominant land-use of the site as fixed effects. Two-way interactions were included between method and log nectar, method and sampling round, log nectar and sampling round, and country and sampling round. All models included an intercept level random effect of sample location (1-5) nested within site (1-14).

This article is protected by copyright. All rights reserved. 
For each FC dataset estimates of abundance for the dominant insect pollinator visitor group were modelled; solitary bees for apples, bumblebees for strawberries and field beans. Data were not sufficient to model the abundance of all groups individually, but models of the total abundance of all bees and hoverflies were run for comparison. Species richness of all bees and hoverflies was also modelled. Initial models included sampling method, the natural log of flower density and their interaction as fixed effects, and an intercept level random effect of sampling section (1-6) nested within site.

\section{Results}

Pan traps and transects implemented by research staff on the wider countryside surveys across $141 \mathrm{~km}$ squares sampled a total of 110 species (16 bumblebee, 38 solitary bee, 55 hoverfly species and the honeybee Apis mellifera) with variations in species richness and abundance for each method (Table 1, Table S3). In the wider countryside, 65\% of solitary bees, $19 \%$ of hoverflies and $14 \%$ of bumblebees recorded by research staff were identified to group level only, because specimens were not netted for identification. Taxonomic experts recorded 10 species of bumblebee, 21 species of solitary bee and 34 species of hoverfly on transects, whilst for the same number of sampling visits to the same transect locations (25, though on different days) research staff recorded 11, nine and 18 species of each respectively. For crops, we recorded a total of 54 species in apples ( 8 bumblebee, 44 solitary bee, 1 hoverfly and the honeybee), 32 species in strawberries (12 bumblebee, 14 solitary bee, 5 hoverfly and the honeybee) and 55 in field beans (14 bumblebee, 31 solitary bee, 9 hoverfly and the honeybee) (Table 2, Table S4 for total species richness and abundance per crop).

\section{Community dissimilarity}

Overall, there was significant dissimilarity between the pollinator communities sampled using pan traps and transects in the wider countryside $\left(\mathrm{R}^{2}=0.121, \mathrm{~F}_{1,24}=3.312, \mathrm{p}<0.001\right)$ driven by more solitary bee and hoverfly species detected by pan traps than transects, but more individuals of common bumblebee species on transects (Figure 2, Table S3, Figure S1a). There was significant dissimilarity between the pollinator communities sampled by pan traps and transects in all crop types; apples $\left(\mathrm{R}^{2}=0.51, \mathrm{~F}_{1,14}=14.309, \mathrm{p}=0.008\right)$; strawberries $\left(\mathrm{R}^{2}=0.29, \mathrm{~F}_{1,14}=5.744, \mathrm{p}=0.008\right)$; field beans $\left(\mathrm{R}^{2}=0.41, \mathrm{~F}_{1,14}=9.58, \mathrm{p}=0.008\right)$. (Figure 3). Transects sampled much higher numbers of bumblebee individuals in strawberries and field beans than did pan traps (around 10 and 5 times respectively, Table S4) with samples more 
dominated by common species than pan traps (Figure S1c-d). In apples were pan traps sampled nearly 10 times the numbers solitary bees (Table S4)

\section{Species accumulation and recorder effects}

For bumblebees in the wider countryside there was close correspondence between the species accumulation rates for each method; although overall pan traps accumulated more species and transects sampled more individuals (Figure 4a). In crops this pattern was accentuated, with the transect method showing lower rates of bumblebee species accumulation per individual sampled and reaching an asymptote, whereas the steeper accumulation curves for pan traps are predicted to continue (Figure 4b). In general the species accumulation curves for bumblebees were broadly similar between pan traps, transects by researchers and transects by taxonomic experts (Figure 5a).

For solitary bees, the same general pattern of species accumulation between pan traps and transects was observed in the wider countryside and in apples and field beans. It was difficult to construct meaningful species accumulation curves for transects (Figure 4c and Figure S2) because a large proportion of individuals were not identified to species resolution (Table S4). However, whilst the number of individuals recorded by taxonomic experts on transects was lower than those sampled in pan traps, species accumulation curves for transects completed by experts suggest that, per individual, this would achieve comparable or better species coverage with greater sampling of individuals (Figure 5b).

Hoverflies were not sampled in crops in high enough numbers, but for the wider countryside rates of species accumulation per individual for pan traps was around double that for transects (Figure 4d). However, it is notable that two species (E. balteatus and S. ribesi) comprised $84 \%$ of individual hoverflies sampled on transects and identifiable to species resolution. Removing these two species leads to greater correspondence between pan taps and transects in species accumulation (Figure S3a). Correspondence between hoverfly species accumulation curves for pan traps and taxonomic experts suggest that they perform comparably in terms of sampling species (Figure 5c). Removing the highly abundant $E$. balteatus and S. ribesis improved the correspondence of researcher transects to expert transects and pan traps (FigureS3b).

This article is protected by copyright. All rights reserved. 
Estimates of abundance for all taxonomic groups were significantly, positively correlated between research staff and volunteers, using transect and focal observations (see supplementary information and Figures S4 and S5 for full results).

\section{Sampling unit level analyses}

There were significant differences between sampling methods in both the abundance and species richness of solitary bees per sampling unit (pan trap station or $200 \mathrm{~m}$ transect section). Pan traps sampled greater numbers of solitary bee individuals $(\beta=-1.27 \pm 0.22, \mathrm{z}=-5.77$, $\mathrm{p}<0.001$; Figure 6b) and species $(\beta=-2.38 \pm 0.27, \mathrm{z}=-8.87, \mathrm{p}<0.001$; Figure $\mathrm{S} 7 \mathrm{~b})$ than transects. However, for bumblebees and hoverflies significant interactions suggest the effects of sampling method on abundance and species richness were dependent on both estimated nectar sugar availability along the $200 \mathrm{~m}$ transect and, for hoverflies, the timing of the sampling round (Tables S5 and S6). On transects, increasing nectar availability had a significant, positive effect compared to pan traps for bumblebee abundance $(\beta=0.28 \pm 0.07$, $\mathrm{z}=4.12, \quad \mathrm{p}<0.001 ;$ Figure 6a) and species richness $(\beta=2.09 \pm 0.34, \quad \mathrm{z}=6.09, \quad \mathrm{p}<0.001$; FigureS7a), and hoverfly abundance $(\beta=0.16 \pm 0.06, z=2.59, p=0.010$; Figure $6 c)$ and species richness $(\beta=0.16 \pm 0.06, z=2.74, p=0.006$; FigureS7c). The effects of country, sampling round and max temperature in the models of abundance and richness are reported in the supplementary material (Tables S5 and S6).

In apples a significant interaction between method and flower density showed a negative effect of increased flower density on solitary bee abundance in pan traps but a positive effect on transects $(\beta=0.87 \pm 0.18, z=4.99, p<0.001$; Figure $7 \mathrm{a})$. The model for abundance of all pollinating insects was qualitatively the same (Table S7), as was that for species richness $(\beta=0.51 \pm 0.13, z=3.92, p<0.001 ;$ Figure S7a, Table S8).

In strawberries, bumblebee abundance on transects was significantly higher than in pan traps regardless of flower density $(\beta=2.27 \pm 0.13, \mathrm{z}=17.00, \mathrm{p}<0.001$; Figure $7 \mathrm{~b})$. However, for the abundance of all pollinating insects, estimates from transects increased significantly with flower density compared to those of pan traps $(\beta=0.52 \pm 0.13, \mathrm{z}=4.10, \mathrm{p}<0.001$; Table S7), as did the number of species sampled $(\beta=0.38 \pm 0.12, z=3.32, p=0.001$; Figure $S 7 b$, Table S8).

This article is protected by copyright. All rights reserved. 
In field beans, a significant interaction between method and flower density showed bumblebee abundance increased with flower density on transects but declined with flower density in pan traps $(\beta=0.38 \pm 0.12, \mathrm{z}=3.32, \mathrm{p}=0.001$; Figure $7 \mathrm{c})$. Results for total pollinator abundance were qualitatively the same $(\beta=0.35 \pm 0.16, \mathrm{z}=2.15, \mathrm{p}=0.032$; Table S7), as were those for the number of species sampled $(\beta=0.42 \pm 0.15, \mathrm{z}=2.88, \mathrm{p}=0.004$; Figure S7c, Table S8).

\section{Discussion}

Understanding the status and trends of pollinators is an urgent global priority requiring development of national-scale monitoring using repeatable and standardised survey methods (Dicks et al., 2016). Our study compared the performance of different pollinator survey methods in sampling different taxonomic groups and when implemented by different recorders varying in experience. We discuss our findings within the context of the logistical and financial constraints presented by large scale biological monitoring.

Pan traps and transects provided a different picture of the pollinating insect community. Overall, the assemblages sampled by the two methods were significantly dissimilar compositionally in both the wider countryside and crop fields; in general, driven by transects sampling fewer species, particularly of solitary bee and hoverfly, but more individual bumblebees, particularly in crops.

Sampling effort dictates the relative performance of methods (Rhoades et al., 2017), for example, increasing the duration of expert transects may result in data that converges on the richness estimates produced by pan-traps. Fundamentally different modes of action make it impossible to properly standardise sampling effort (e.g. sampling duration) between pan traps and transects. However, using species accumulation curves, we were able to compare estimates of species richness produced by the different methods and actors to understand the extent that sampling effort (i.e. numbers of individuals collected) contributes to the observed differential patterns. Accumulation of species occurring at a similar rate indicates that differences in relative sampling effort are driving differences in species richness. We found higher species accumulation rates for pan traps, except for bumblebees in the wider countryside, suggesting factors other than sample size are driving differences between methods.

This article is protected by copyright. All rights reserved. 
In all datasets, transects sampled more individual bumblebees than pan traps, probably due in part to the strong positive association between floral resources and bumblebee counts on transects and to the bias in pan traps against sampling larger bodied insects (Cane et al., 2000). That this difference was of a greater magnitude in strawberry and field bean fields compared to the wider countryside may be because these crops are predominantly bumblebee pollinated (Kleijn et al., 2015) and due to the competition for bumblebee visits from the abundant floral displays of these crop monocultures lowering pan trap catches. However, pan traps showed higher rates of species accumulation and generally sampled more species of bumblebee. One explanation is that the transect protocol was constrained to record flower visitors only, so species foraging specialism will reduce the pool of species being sampled, particularly in crops (where only one flower type was surveyed).

For solitary bees, pan traps collected more species and individuals than transects, and in apples the larger magnitude of difference in numbers collected may relate to the 24-hour pan trapping used (as opposed to 6-7 hours). Projecting species accumulation was difficult for transects due to low rates of species level identification. However, when experts undertook transects in the wider countryside, though the number of solitary bees recorded was still lower than pan traps, species accumulation rate per individual became higher for transects. These findings highlight a limitation when using such "real-time" methods to collect data on solitary bees that are difficult to detect, identify or capture, particularly for less experienced recorders. For hoverflies, pan traps showed similarly higher rates of species accumulation per individual sampled than transects, but again, expert recorders mitigated this by providing a convergent rates of species accumulation between methods.

Whilst expertise seems necessary to collect species resolution data from transects, our results suggest transects could be suitable for novices to collect group level abundance data of bumblebees and possibly hoverflies, with basic instructions. However, we found the potential for miscounts or misclassifications, particularly for hoverflies. Kremen et al (2011), similarly found estimates of bee abundance were correlated between volunteers with five hours training and experts. A transect based (1-2km) approach in 373 sites, 'BeeWalks', has been developed by the Bumblebee Conservation Trust in the UK and is generating data on trends in abundance for bumblebee species (Comont \& Dickinson, 2017). However, training, 
assessment and data validation processes are needed before mass participation observational methods are widely adopted for monitoring.

Across all surveys, per sampling unit estimates of abundance and species richness on transects increased with estimated nectar availability or floral density. This effect is intrinsic to the method (transects recorded flower visitors), but the strength of response for different taxonomic groups to floral resources may reflect their different ecologies. Social bumblebees increase colony foraging activity in response to nectar availability (Dornhaus \& Chittka, 2001) and over larger ranges than smaller, solitary bee species (Osborne et al., 1999; Gathmann \& Tscharntke, 2012). This may explain the strong response of bumblebees to transect floral resources in the wider countryside compared with solitary bees that possess smaller foraging ranges and a lack of social recruitment behaviour. Hoverflies also do not recruit, but are not restricted to foraging around nest sites, and so individuals may freely aggregate around high floral resources. This is consistent with our results showing a positive relation between hoverfly abundance and nectar availability.

For transects, abundance records may reflect population densities in a location but also the redistribution of individuals across a landscape in response to temporary increases in floral resources (Carvell et al 2015); however methods are now available to address this (Kleijn et al., 2018). The negative relationships between local floral density and the number of individuals (and species) caught in pan traps in flowering crop fields suggest that crop flowers were 'competing' with pan traps by drawing away insects (e.g. Cane et al., 2000). If pan trapping is confounded by floral densities, this could affect their use in monitoring schemes as it may lead to erroneous detection of declines if an areas floral resources increase over time. However, this inverse relationship between pan trap catch and floral density was particular to crops, likely due to the very high flower densities in these crop monocultures. The magnitude of floral 'competition' with pan traps will be lower in florally heterogeneous wider countryside environments. Moreover, our results reflect a series of snaphot samples of the different methods in space. Structured, longitudinal monitoring or experiments manipulating floral densities are needed to demonstrate how pan trap catches might respond to annual and multiannual changes in floral resources at a given site. It must be noted that our nectar estimates and pan trap stations were not precisely spatial coincident and quantifying floral resources in a fixed area surrounding the pan traps (in the wider countryside setting) may have given different results (Carvell et al., 2016). Previous findings on the impacts of 
floral resources on pan trap catches have also been mixed; with negative effects on abundance (Roulston, Smith, \& Brewster, 2007) and species richness (Baum \& Wallen, 2011), positive effects on abundance (e.g. Wood et al., 2015), and no effect (e.g. Rhoades et al., 2017). Overall, measures accounting for local floral resources will be a vital covariate for collection with any method used in pollinator survey protocols for monitoring.

Pan traps and transects have different utility and efficacy for monitoring different aspects of pollinator biodiversity. Identifying the objective of the monitoring and what metrics of the pollinator community are required is essential to determining which methods are employed. Characterising plant-pollinator interactions or identifying which species of insect are delivering pollination service to crops and wildflowers require transects (or other observational methods) as pan traps do not reflect this (Kleijn et al., 2015; Gibbs et al., 2017). While pan traps have limitations and biases, they provide species resolution data independent of expertise and require less person effort to achieve equivalent sample sizes when compared to transects. They could also minimise noise in the data from different levels of recorder knowledge or changes in recorders over time. Our results show that, independent of differences in sampling effort, transects conducted by people without a large degree of taxonomic expertise will not sample the same number of species as pan traps, and for solitary bees they require considerably more sampling effort to detect as many individuals. This could be particularly important when recorders with appropriate expertise are a limiting factor, along with logistical and resourcing implications. For example, if species-level abundance and diversity of solitary bees were targeted, our results suggest five transects would require sampling for 36 - 45 minutes by someone with extensive experience and taxonomic expertise to achieve equivalent sample sizes and species coverage as five 6-7 hours of pan traps. If staff availability or resources are limiting, pan traps using non-expert recorders coupled with species identification by experts can be used (Le Féon et al., 2016) and molecular methods may soon be an option (Creedy et al., 2019). Though lethal, pan traps are unlikely to reduce pollinating insect populations at the sampling intensities tested here (Gezon et al., 2015).

No one sampling method can fully characterise the pollinating insect community at a given location, but sampling should aim to provide necessary taxonomic coverage and keep bias as consistent as possible over time. Furthermore, combining data from different locations requires methods that ensure datasets are at least comparable at their most basic resolution. A national pollinator monitoring scheme could employ pan traps and observational methods to

This article is protected by copyright. All rights reserved. 
allow the complimentary recording of different facets of the pollinator community including abundance, species richness, functional roles and pollination service potential. A crucial caveat, however, is the differential effect of local floral resource availability on the efficacy of the pan traps and observational methods and how this may influence the data obtained and the conclusions drawn. This potential complementarity and caveat should both be considered carefully during method(s) selection alongside monitoring objectives, desired metrics and the availability of financial or human resources. Only through such standardardisation can monitoring efforts become internationally cohesive. The value of obtaining standardised datasets on pollinating insects cannot be overstated in providing robust evidence on longterm and large-scale patterns and trends to inform national and international policy needs.

\section{Acknowledgements}

The UK Department for the Environment, Food and Rural Affairs, the Scottish Government and the Welsh Government funded the wider countryside survey under project WC1101. The crops surveys were funded jointly by grant BB/I000348/1 from BBSRC, Defra, NERC, the Scottish Government and the Wellcome Trust, under the Insect Pollinators Initiative. This work was supported by the Natural Environment Research Council award number NE/R016429/1, part of the UK-SCAPE programme delivering National Capability. Thanks to the farmers, land owners and land managers who allowed us access to their land. Thanks to A. Perry, D. Chapman, N. Majlessi, A. Turner, D. Coston, C. Dodson, R. Evans, L. Truslove and M. Lappage for undertaking fieldwork and to all the non-expert volunteers. Thanks to $\mathrm{S}$. Freeman for statistical advice. Thanks to three reviewers whos insights and suggestions improved the manuscript.

\section{Authors Contribution's}

$\mathrm{RO}-\mathrm{HR}, \mathrm{AV}$, and $\mathrm{CC}$ concieved and designed the project. RO, CA-MH and SR-CC collected and collated the wider countryside data, and MH-IW provided specimen identifications. MG coordinated the collection of and provided the flowering crop data. RO analyzed the data. RO-HR, AV and CC led the writing of the manuscript. All authors contributed critically to drafts and gave final approval for publication. 


\section{Data Accessibility}

Data for the wider countryside surveys are available from the NERC Environmental Information Data Centre: https://doi.org/10.5285/69a0d888-9f6b-4e67-8d29-402af1412d8e. Data for the flowering crops surveys are available from Dryad repository; http://datadryad.org/resource/10.5061/dryad.31f7ph7.

\section{References}

Baude, M., Kunin, W. E., Boatman, N. D., Conyers, S., Davies, N., Gillespie, M. A. K., ... Memmott, J. (2016). Historical nectar assessment reveals the fall and rise of floral resources in Britain. Nature, 530, 80-85. doi:DOI: 10.1038/nature16532

Baum, K. A., \& Wallen, K. E. (2011). Potential Bias in Pan Trapping as a Function of Floral Abundance. Journal of the Kansas Entomological Society, 84(2), 155-159. doi:10.2317/jkes100629.1

Cameron, S. A., Lozier, J. D., Strange, J. P., Koch, J. B., Cordes, N., Solter, L. F., ... Robinson, G. E. (2011). Patterns of widespread decline in North American bumble bees. Proceedings of the National Academy of Sciences of the United States of America, 108(2), 662-667. doi:10.1073/pnas.1014743108

Cane, J. H., Minckley, R. L., \& Kervin, L. J. (2000). Sampling Bee (Hymenoptera: Apiformes) for pollinator community studies: Pitfall of Pan-Trapping. Journal of the Kansas Entomological Society, 73(4), 225-231.

Carvalheiro, L. G., Kunin, W. E., Keil, P., Aguirre-Gutiérrez, J., Ellis, W. N., Fox, R., ... Biesmeijer, J. C. (2013). Species richness declines and biotic homogenisation have slowed down for NW-European pollinators and plants. Ecology Letters, 16(7), 870-878. doi:10.1111/ele.12121

Carvell, C., Bourke, A. F. G., Osborne, J. L., \& Heard, M. S. (2015). Effects of an agrienvironment scheme on bumblebee reproduction at local and landscape scales. Basic and Applied Ecology, 16(6), 519-530. doi:10.1016/j.baae.2015.05.006

Carvell, C., Isaac, N. J. B., Jitlal, M., Peyton, J., Powney, G. D., Roy, D. B., .. Roy, H. E. (2016). Design and Testing of a National Pollinator and Pollination Monitoring Framework. Final summary report to the Department for Environment, Food and Rural Affairs (Defra), Scottish Government and Welsh Government: Project WC1101.

Comont, R. F., \& Dickinson, H. (2017). BeeWalk Annual Report 2017. Retrieved from https://bumblebeeconservation.org/images/uploads/Beewalk/BBCT074_- 
_BeeWalk_Annual_Report_2017_03.17_(1).pdf

Creedy, T. J., Norman, H., Tang, C. Q., Chin, K. Q., Andujar, C., Arribas, P., ... Vogler, A. P. (2019). A validated workflow for rapid taxonomic assignment and monitoring of a national fauna of bees (Apiformes) using high throughput DNA barcoding. Molecular Ecology Resources, Advance on. doi:10.1111/1755-0998.13056

Dennis, R. L. H., Shreeve, T. G., Isaac, N. J. B., Roy, D. B., Hardy, P. B., Fox, R., \& Asher, J. (2006). The effects of visual apparency on bias in butterfly recording and monitoring. Biological Conservation, 128(4), 486-492. doi:10.1016/j.biocon.2005.10.015

Dicks, L. V., Viana, B., Bommarco, R., Brosi, B., Arizmendi, C., Cunningham, S. A., ... Taki, H. (2016). Ten policies for pollinators: What governments can do to safeguard pollination services. Science, 354(6315), 14-15. doi:10.1126/science.aai9226

Dornhaus, A., \& Chittka, L. (2001). Food alert in bumblebees (Bombus terrestris): Possible mechanisms and evolutionary implications. Behavioral Ecology and Sociobiology, 50(6), 570-576. doi:10.1007/s002650100395

Garratt, M. P. D., \& Potts, S. G. (2011). Data from: Monitoring insect pollinators and flower visitation: the effectiveness and feasibility of different survey methods. Methods in Ecology and Evolution. doi:10.5061/dryad.31f7ph7.

Gathmann, A., \& Tscharntke, T. (2012). Foraging ranges of solitary bees. Journal of Animal Ecology, 71(5), 757-764. doi:10.1046/j.1365-2656.2002.00641.x

Gezon, Z. J., Wyman, E. S., Ascher, J. S., Inouye, D. W., \& Irwin, R. E. (2015). The effect of repeated, lethal sampling on wild bee abundance and diversity. Methods in Ecology and Evolution, 6(9), 1044-1054. doi:10.1111/2041-210X.12375

Gibbs, J., Joshi, N. K., Wilson, J. K., Rothwell, N. L., Powers, K., Haas, M., ... Isaacs, R. (2017). Does passive sampling accurately reflect the bee (apoidea: Anthophila) communities pollinating apple and sour cherry orchards? Environmental Entomology, 46(3), 579-588. doi:10.1093/ee/nvx069

Hsieh, T. C., Ma, K. H., \& Chao, A. (2019). iNEXT: Interpolation and Extrapolation for Species Diversity.

Isaac, N. J. B., \& Pocock, M. J. O. (2015). Bias and information in biological records. Biological Journal of the Linnean Society, 115(3), 522-531. doi:10.1111/bij.12532

Kleijn, D., Linders, T. E. W., Stip, A., Biesmeijer, J. C., Wäckers, F. L., \& Bukovinszky, T. (2018). Scaling up effects of measures mitigating pollinator loss from local- to landscape-level population responses. Methods in Ecology and Evolution, 9(7), 17271738. doi:10.1111/2041-210X.13017

This article is protected by copyright. All rights reserved. 
Kleijn, D., Winfree, R., Bartomeus, I., Carvalheiro, L. G., Henry, M., Isaacs, R., ... Potts, S. G. (2015). Delivery of crop pollination services is an insufficient argument for wild pollinator conservation. Nature Communications, 6(May), 7414. doi:10.1038/ncomms8414

Kremen, C., Ullman, K. S., \& Thorp, R. W. (2011). Evaluating the quality of citizen-scientist data on pollinator communities. Conserv Biol, 25(3), 607-617. doi:10.1111/j.15231739.2011.01657.x

Le Féon, V., Henry, M., Guilbaud, L., Coiffait-Gombault, C., Dufrêne, E., Kolodziejczyk, E., ... Vaissiière, B. E. (2016). An expert-assisted citizen science program involving agricultural high schools provides national patterns on bee species assemblages. Journal of Insect Conservation, 20(5), 905-918. doi:10.1007/s10841-016-9927-1

Lebuhn, G., Droege, S., Connor, E. F., Gemmill-Herren, B., Potts, S. G., Minckley, R. L., ... Parker, F. (2013). Detecting Insect Pollinator Declines on Regional and Global Scales. Conservation Biology, 27(1), 113-120. doi:10.1111/j.1523-1739.2012.01962.x

LeBuhn, G., Droege, S., Connor, E., Gemmill-Herren, B., \& Azzu, N. (2016). Protocol to detect and monitor pollinator communities. Guidance for practitioners.

Lenth, R. V. (2016). Least-Squares Means: The R Package lsmeans. Journal of Statistical Software, 69(1), 1-33.

Lüdecke, D. (2017). Data Visualization for Statistics in Social Science.

O’Connor, R. S., Jones, C. M., Carvell, C., Peyton, J., Vanbergen, A. J., Andrews, C., \& Kunin, W. E. (2016). Data from: Monitoring insect pollinators and flower visitation: the effectiveness and feasibility of different survey methods. Methods in Ecology and Evolution. doi:10.5285/69a0d888-9f6b-4e67-8d29-402af1412d8e

Oksanen, J., Blanchet Guillaume., F., Kindt, R., Legendre, P., Minchin, P. R., O’Hara, R. B., ... Wagner, H. (2015). vegan: Community Ecology Package. R package version 2.3-1. Retrieved from http://cran.r-project.org/package=vegan

Osborne, A. J. L., Clark, S. J., Morris, R. J., Williams, I. H., Riley, J. R., Smith, a D., ... Edwards, a S. (1999). A landscape-scale of bumble bee foraging study range and constancy using harmonic radar. Journal of Applied Ecology, 36(4), 519-533. doi:10.1046/j.1365-2664.1999.00428.x

Pescott, O. L., Walker, K. J., Pocock, M. J. O., Jitlal, M., Outhwaite, C. L., Cheffings, C. M., ... Roy, D. B. (2015). Ecological monitoring with citizen science: The design and implementation of schemes for recording plants in Britain and Ireland. Biological Journal of the Linnean Society, 115(3), 505-521. doi:10.1111/bij.12581

This article is protected by copyright. All rights reserved. 
Pinheiro, J., Bates, D., DebRoy, S., Sarkar, D., \& Team., R. C. D. (2015). Linear and nonlinear mixed effects models. $\mathrm{R}$ package version 3.1-122.

Potts, S. G., Imperatriz-Fonseca, V., Ngo, H. T., Aizen, M. A., Biesmeijer, J. C., Breeze, T. D., ... Vanbergen, A. J. (2016). Safeguarding pollinators and their values to human well-being. Nature, 540(7632), 220-229. doi:10.1038/nature20588

Powney, G. D., Carvell, C., Edwards, M., Morris, R. K. A., Roy, H. E., Woodcock, B. A., \& Isaac, N. J. B. (2019). Widespread losses of pollinating insects in Britain. Nature Communications, 10(1), 1018. doi:10.1038/s41467-019-08974-9

R Core Team. (2016). R: A Language and Environment for Statistical Computing. Vienna, Austria: R Foundation for Statistical Computing.

Rhoades, P., Griswold, T., Waits, L., Bosque-Pérez, N. A., Kennedy, C. M., \& Eigenbrode, S. D. (2017). Sampling technique affects detection of habitat factors influencing wild bee communities. Journal of Insect Conservation, O(0), 0. doi:10.1007/s10841-0170013-0

Roulston, T., Smith, S. A., \& Brewster, A. L. (2007). A comparison of Pan Trap and Intensive Net Sampling Techniques for Documentung a Bee (Hymenoptera: Apiformes) Fauna. Journal of the Kansas Entomological Society, 80(2), 179-181.

Skaug, H., Fournier, D., Bolker, B., Magnusson, A., \& Nielsen, A. (2015). glmmADMB A Generalized Linear Mixed Models using 'AD Model Builder'.. R package version 0.8.3.2.

Sutherland, W. J., Roy, D. B., \& Amano, T. (2015). An agenda for the future of biological recording for ecological monitoring and citizen science. Biological Journal of the Linnean Society, 115(3), 779-784. doi:10.1111/bij.12576

Vanbergen, A. J., Baude, M., Biesmeijer, J. C., Britton, N. F., Brown, M. J. F., Brown, M., ... Wright, G. A. (2013). Threats to an ecosystem service: pressures on pollinators. Frontiers in Ecology and the Environment, 11(5), 251-259. doi:10.1890/120126

Westphal, C., Bommarco, R., Carre, G., Lamborn, E., Morison, N., Petanidou, T., ... SteffanDewenter, I. (2008). Measuring bee diversity in different habitats European habitats and Biogeographical Regions. Ecological Monographs, 78(4), 653-671. doi:10.1890/071292.1

Wood, T. J., Holland, J. M., \& Goulson, D. (2015). A comparison of techniques for assessing farmland bumblebee populations. Oecologia, 177(4), 1093-1102. doi:10.1007/s00442015-3255-0

This article is protected by copyright. All rights reserved. 
Zuur, A. F., Hilbe, J. M., \& Ieno, E. N. (2013). A Beginner's Guide to GLM and GLMM with R: A Frequentist and Bayesian Perspective for Ecologists. Highland Statistics.

Table 1 Mean \pm SE abundance and species richness per sampling site $(n=14)$ sampled by research staff across the wider countryside.

\begin{tabular}{llllllll}
\hline & Abundance & & \multicolumn{4}{l}{ Species richness } \\
\hline Method & Bumblebee & Solitary bee & Honeybee & Hoverfly & Bumblebee & $\begin{array}{l}\text { Solitary } \\
\text { bee }\end{array}$ & Hoverfly \\
& & & & & & & \\
\hline Pan Trap & $12.14 \pm 3.17$ & $18.36 \pm 5.77$ & $3.00 \pm 1.03$ & $32.07 \pm 7.53$ & $2.36 \pm 0.59$ & $2.43 \pm 0.74$ & $9.43 \pm 1.28$ \\
Transect & $17.86 \pm 3.18$ & $5.86 \pm 2.35$ & $4.36 \pm 1.39$ & $39.79 \pm 16.93$ & $2.64 \pm 0.42$ & $0.5 \pm 0.24$ & $3.64 \pm 0.75$ \\
\hline
\end{tabular}

This article is protected by copyright. All rights reserved. 
Table 2 Mean abundance \pm SE and species per sampling site for apples, strawberry and field bean sites.

\begin{tabular}{lllllllll}
\hline & & Abundance & & & & \multicolumn{3}{l}{ Species } \\
\hline Crop & Method & Bumblebee & Solitary bee & Honeybee & Hoverfly & Bumblebee & Solitary bee & Hoverfly \\
\hline Apple & Pan trap & $2.63 \pm 0.46$ & $148.88 \pm 53.82$ & $0.88 \pm 0.35$ & $0.13 \pm 0.13$ & $2.25 \pm 0.53$ & $16.88 \pm 2.22$ & $0.13 \pm 0.13$ \\
& Transect & $4.38 \pm 0.98$ & $14.00 \pm 3.49$ & $5.88 \pm 1.64$ & $1.38 \pm 1.10$ & $2.13 \pm 0.40$ & $2.00 \pm 0.38$ & $0.00 \pm 0.00$ \\
Strawb & Pan trap & $15.75 \pm 6.01$ & $11.13 \pm 2.75$ & $5.25 \pm 2.02$ & $3.75 \pm 1.29$ & $3.75 \pm 0.53$ & $4.13 \pm 0.81$ & $0.88 \pm 0.23$ \\
& Transect & $147.25 \pm 32.28$ & $1.75 \pm 0.65$ & $121.00 \pm 34.55$ & $40.00 \pm 12.30$ & $3.88 \pm 0.35$ & $0.38 \pm 0.26$ & $0.25 \pm 0.16$ \\
FieldB & Pan trap & $16.50 \pm 6.35$ & $33.75 \pm 4.55$ & $3.50 \pm 1.58$ & $2.38 \pm 0.46$ & $4.63 \pm 0.84$ & $12.25 \pm 0.88$ & $1.63 \pm 0.26$ \\
& Transect & $65.38 \pm 9.43$ & $1.88 \pm 0.58$ & $8.75 \pm 1.96$ & $1.25 \pm 0.45$ & $5.63 \pm 0.38$ & $0.88 \pm 0.30$ & $0.13 \pm 0.13$ \\
\hline
\end{tabular}

Figure 1 a) Distribution of study sites, showing the agricultural wider countryside sites (brown circles). semi-natural wider countryside sites (yellow circles), strawberry sites (red stars), field bean sites (red squares) and apple sites (red triangles); b) The layout of pan traps and transects for the wider countryside 'one-day' protocol at a $1 \mathrm{~km}$ sampling square; c) The layout of pan traps and transects in a sampling plot for flowering crops.

Figure 2 Non-metric multi-dimensional scaling (NDMS) plot of pan traps (larger dark grey circles) and transects (larger light grey circles) for all species of bee and hoverfly detected in the wider countryside by non-expert researchers. Bumblebee are shown by stars, Apis mellifera a square, solitary bees by triangles and hoverflies by circles. Circles with the same number are for the same site and the polygons connecting sites indicate the overlap between samples.

Figure 3 Non-metric multi-dimensional scaling (NDMS) plots of pan traps (larger dark grey circles) and transects (larger light grey circles) for all species of bee and hoverfly detected in a) apples, b) strawberries and c) field beans. Bumblebee are shown by stars, Apis mellifera a square, solitary bees by triangles and hoverflies by circles. Circles with the same number are for the same site and the polygons connecting sites indicate the overlap between samples.

Figure 4 Individual based species accumulation curves across the whole datasets pooled for a) bumblebees in the wider countryside b) bumblebees in field beans and strawberries c) solitary bees in the wider countryside and d) hoverflies in the wider countryside. Curves were plotted based on data grouped across all sites, using the iNEXT package in R. The solid line shows predications based on interpolation and the dashed part shows predictions based on extrapolation. $95 \%$ confidence intervals are shown as shaded areas.

Figure 5 Individual based species accumulation curves from a subset of data from across 7 of the wider country sites providing corresponding data from pan traps, transects conducted by researcher and transects conducted by professional experts for a) bumblebees, b) solitary bees and c) hoverflies. The solid line shows predictions based on interpolation dashed line the predictions based on extrapolation. 95\% confidence intervals are shown as shaded areas.

This article is protected by copyright. All rights reserved. 
Figure 6 Plots showing predictions from the wider countryside of a) the marginal effects of sampling method and nectar sugar availability on bumblebee abundance b) the least square mean per method for solitary bee abundance and c) predictions of the marginal effects of sampling method and nectar sugar availability on hoverfly abundance. Unbroken lines show predicted values for pan traps and broken for transects. 95\% confidence intervals are shown in grey. Error bars on points show \pm SE. The sampling unit for pan traps is a trapping station (triplet of bowls) and for transects is a 200m section (Figure 1b). Model results are presented in Table S4. Models for species richness are presented in Figure 4S and Table S5.

Figure 7 Plots showing a) predictions for marginal effects of sampling method and flower density on solitary bee abundance in apple crops b) mean abundance bumblebee per sampling method in strawberry crops and c) predictions for marginal effects of sampling method and flower density on bumblebee abundance in field bean crops. Unbroken lines show predicted values for pan traps and broken for transects. 95\% confidence intervals are shown in grey. Error bars on points show \pm SE. Sampling unit for pan traps is a trapping station (triplet of bowls) and for transects is a 50m section (Figure1c). Model results are presented in Tables S7. Models for the species richness of all bees and hoverflies are shown in Figure S5 and Table S8.

This article is protected by copyright. All rights reserved. 


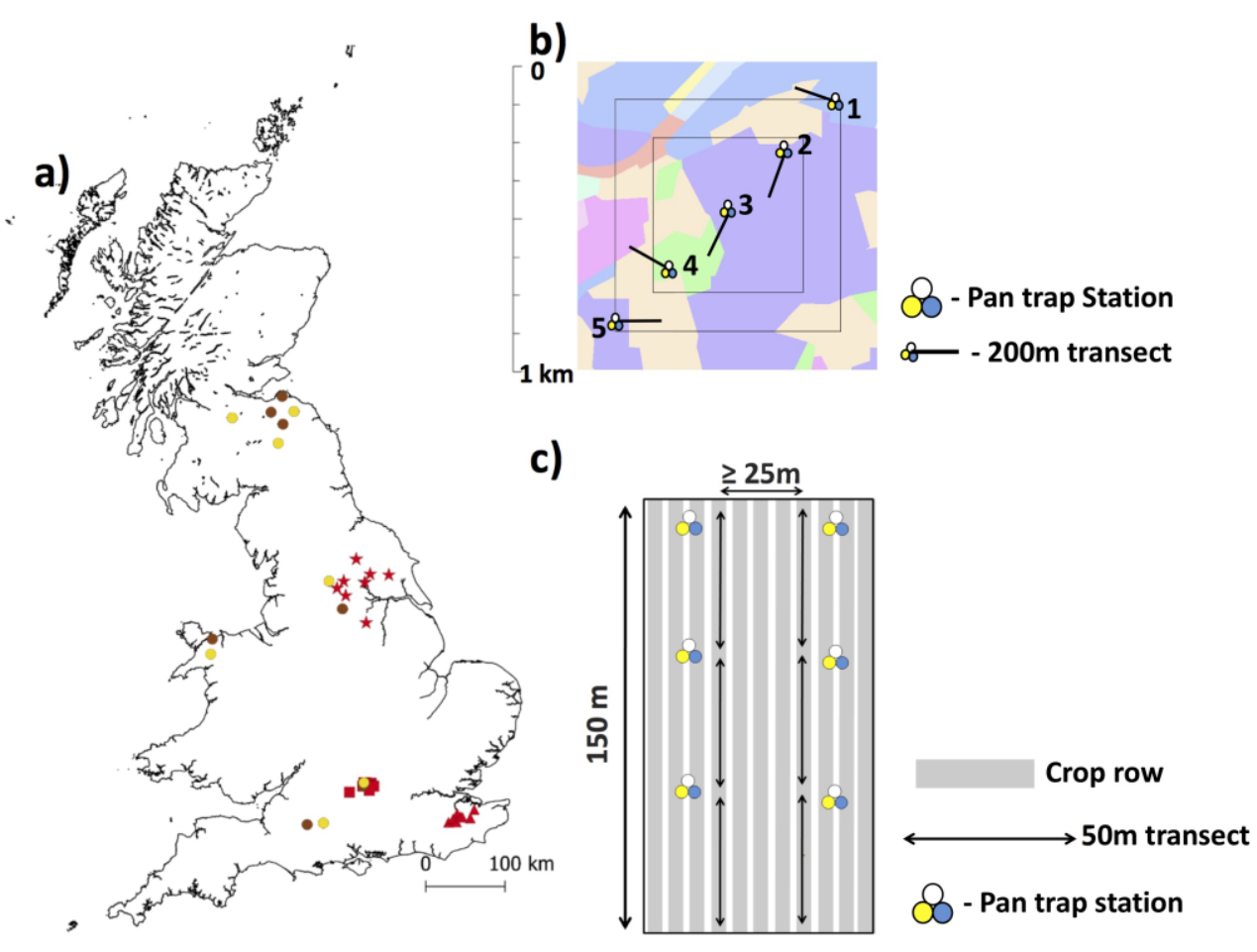

This article is protected by copyright. All rights reserved. 


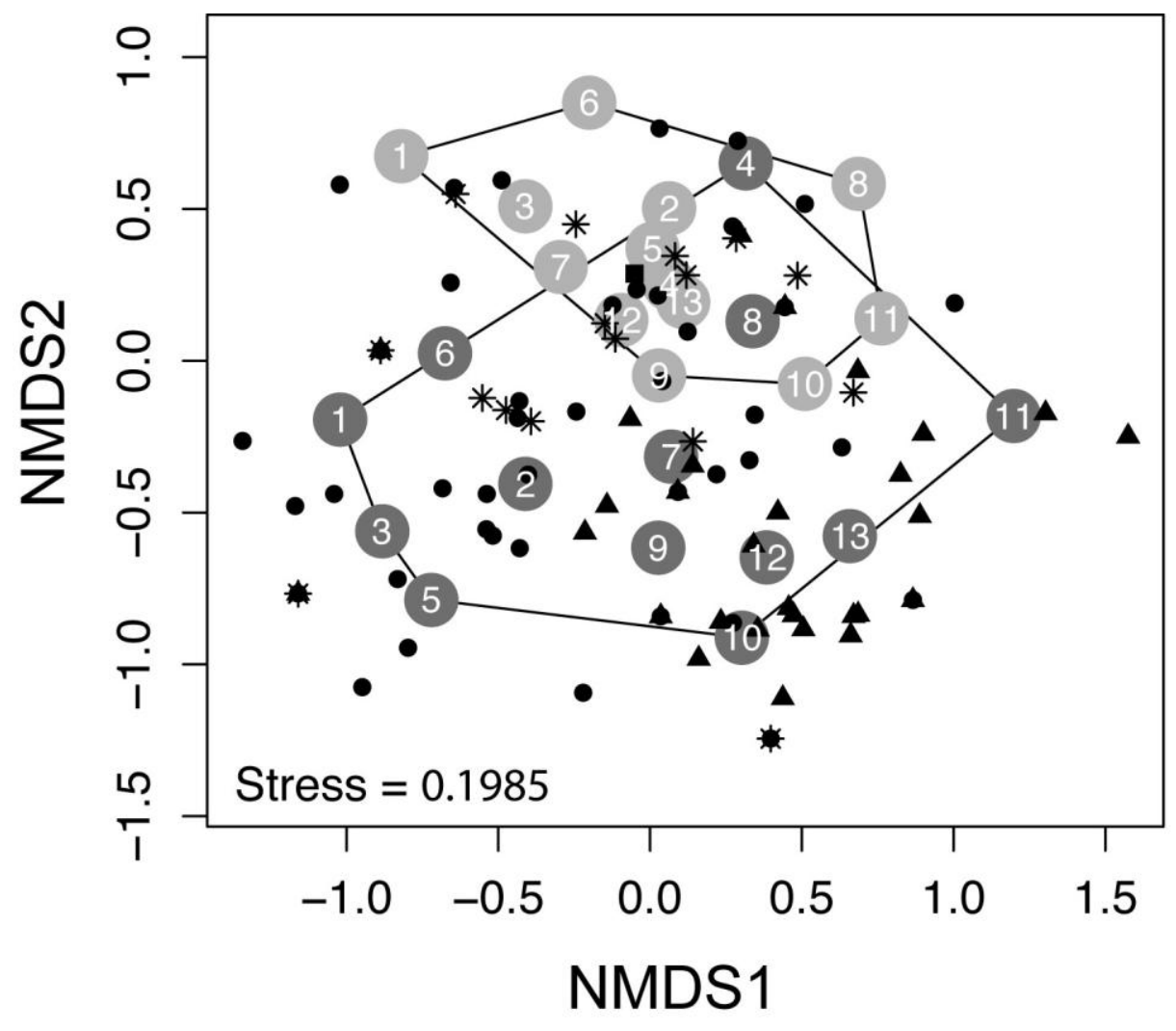

This article is protected by copyright. All rights reserved. 

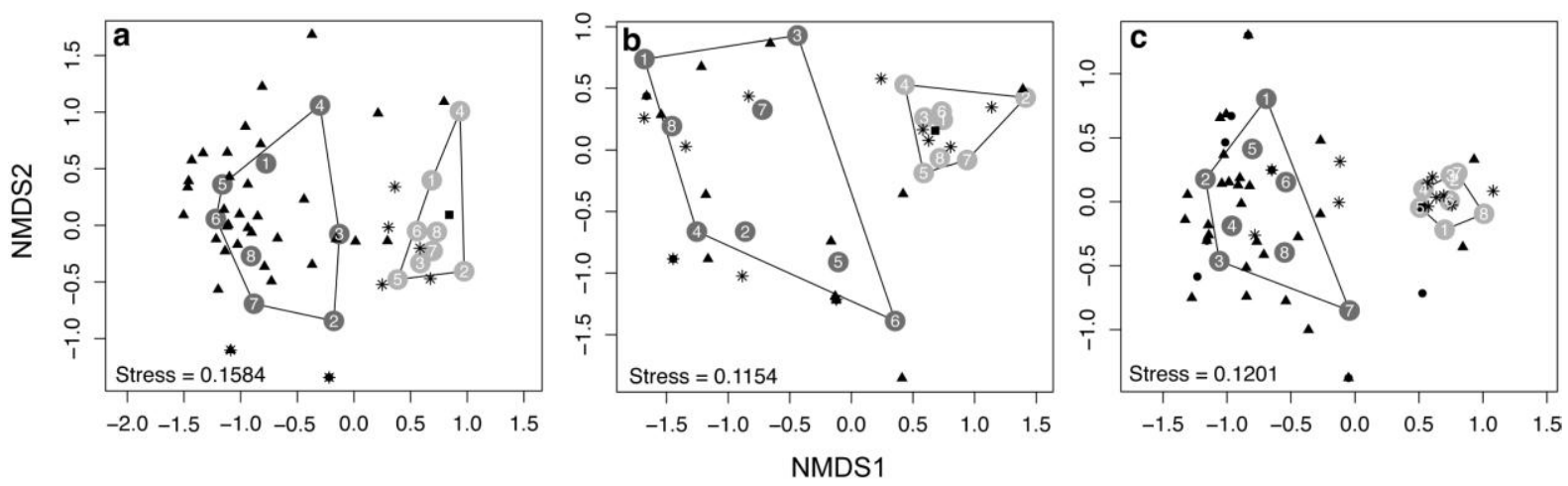

This article is protected by copyright. All rights reserved. 

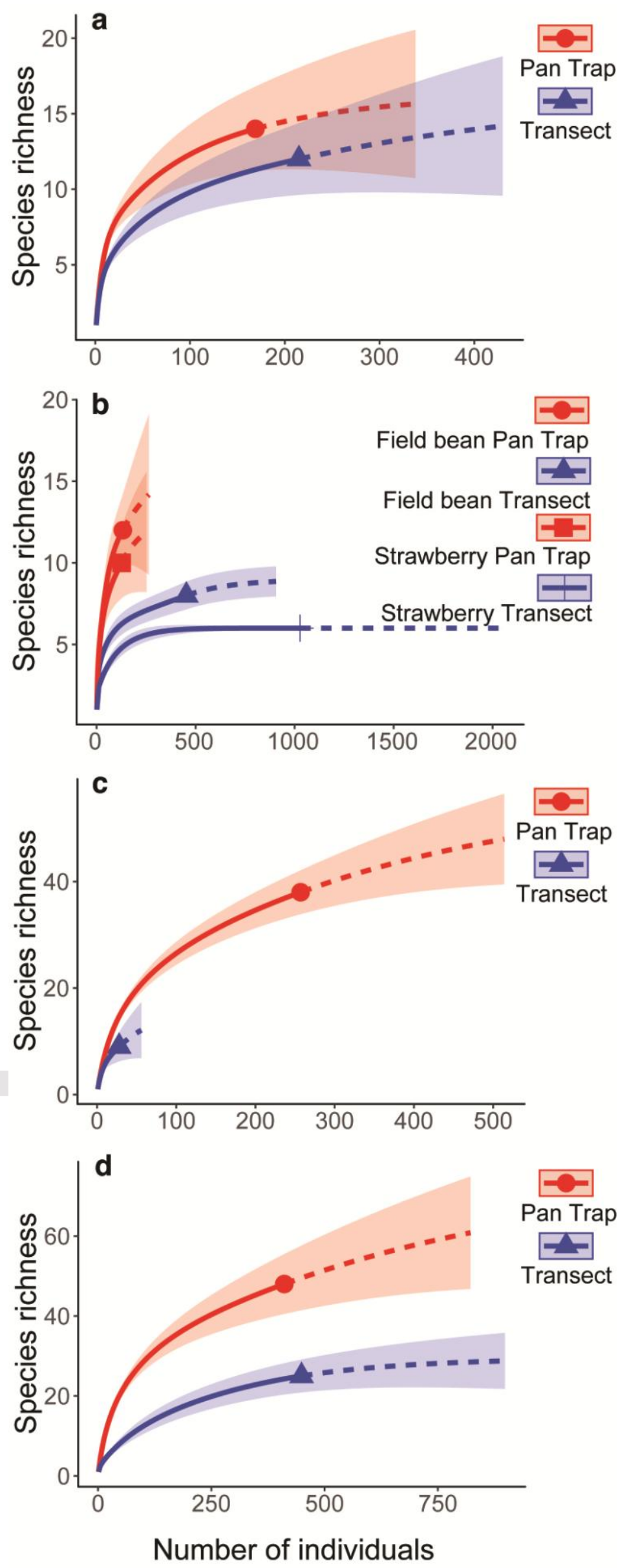

This article is protected by copyright. All rights reserved. 

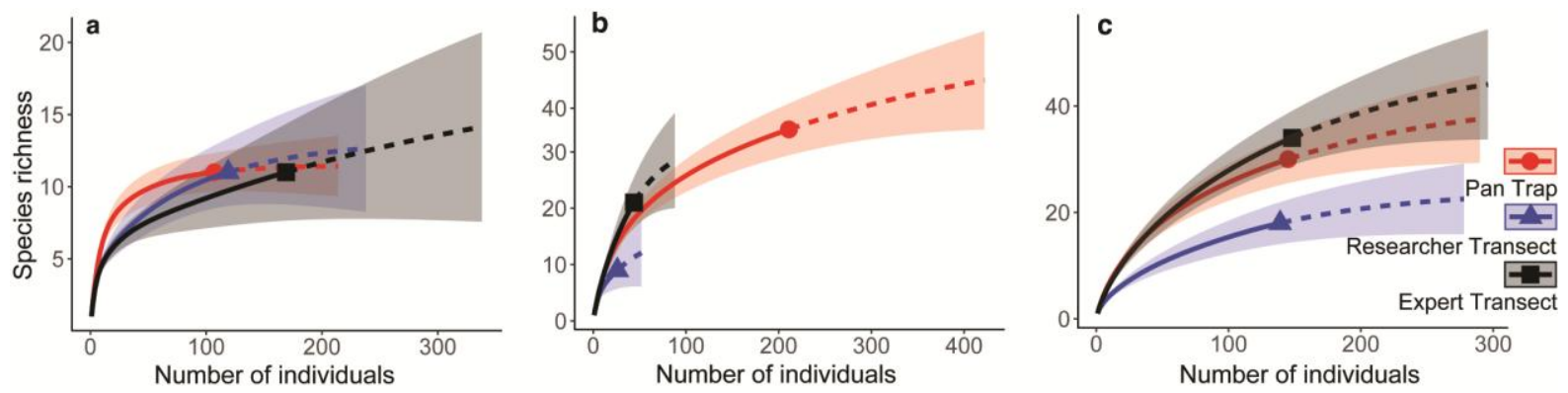

This article is protected by copyright. All rights reserved. 

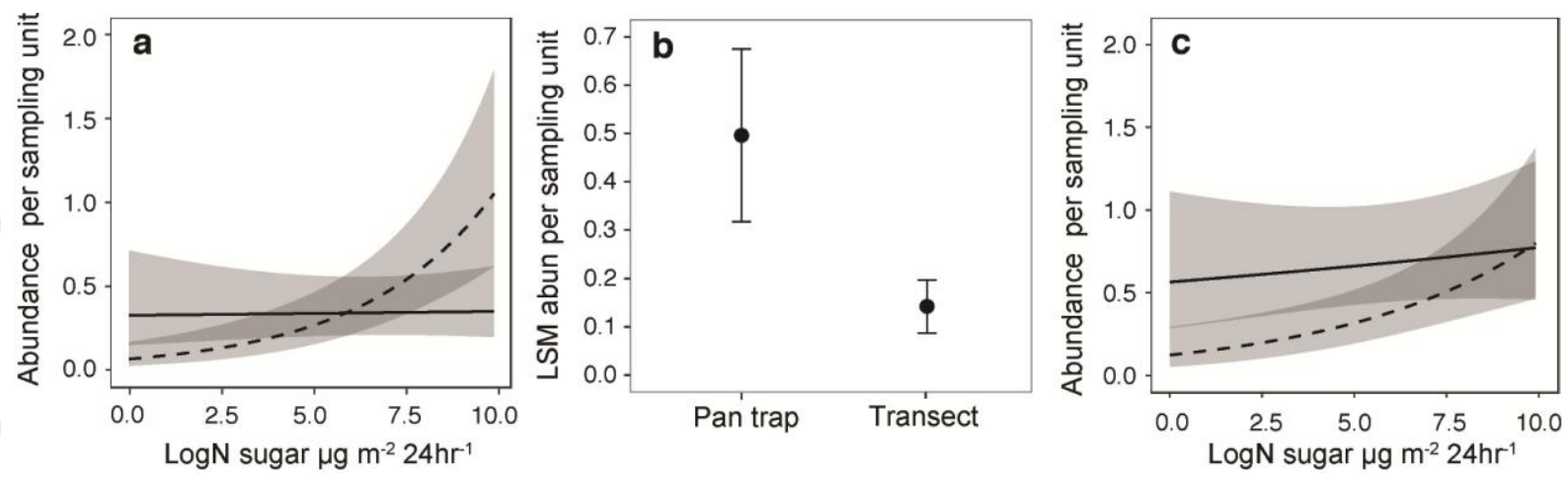

This article is protected by copyright. All rights reserved. 

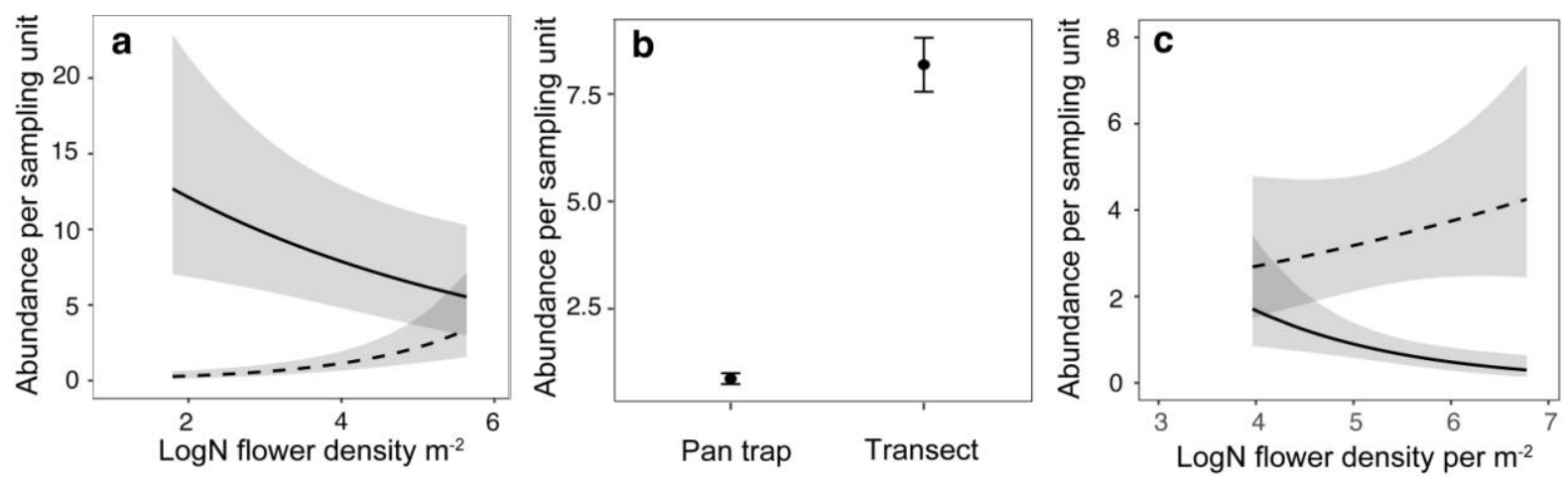

This article is protected by copyright. All rights reserved. 